\title{
Impact of Crosstalk on the Performance of Multi-Wavelength Optical Cross-connected Networks using Deflection Routing
}

\author{
G. A. Castanon, O. K. Tonguz and A. Bononi ${ }^{1}$.
}

\begin{abstract}
BER performance of multi-hop transparent optical networks in uniform traffic under hot-potato, singlebuffer deflection routing schemes is presented. Device-induced crosstalk and ASE noise, limit the characteristics of the network, such as propagation distance, sustainable traffic, and bit-rate.
\end{abstract}

\section{INTRODUCTION}

Optical crosstalk imposes a major limit to the practical implementation of multiwavelength optical crossconnected networks. It is therefore important to know how crosstalk scales with network size and traffic load. The traffic performance of multi-hop packet-switching networks such as Manhattan Street Network (MS) and ShuffleNet (SN) has been studied extensively. However, relatively few studies consider the BER performance of these networks. In [2] ultrafast soliton communication is used to evaluate the BER in multihop networks with deflection routing, but the impact of crosstalk is not considered. In [3] the BER is analyzed using a semi-analytical simulation method for estimating the effect of the interference noises on the BER performance of a circuit switching network. However, the impact of traffic randomness is not considered in the analysis.

This paper presents the first complete BER analysis based on the traffic randomness of multi-hop packetswitched transparent multiwavelength networks. It is shown that the BER performance strongly depends on the traffic load of the network. We present the limit of operation based on a uniform traffic scenario. The main impairments considered in the analysis are intra-band crosstalk and ASE noise.

\section{ANALYSIS}

Fig. 1 shows the node composed of a stack of submodules, one per wavelength. The wavelengths from the input fibers are spatially demultiplexed and sent to the appropriate submodule for add/drop operations and switching. Packets from the submodules are finally re-multiplexed onto the output fibers. The submodule is described in [2].

The intra-band crosstalk generated by the $2 \times 2$ space switch and the one produced by the combination of the demultiplexer (DEMUX) and multiplexer

\footnotetext{
1 The authors are with the Photonics Research Laboratory, Department of Electrical and Computer Engineering, State University of New York at Buffalo, Buffalo, NY 14260.
}

(MUX) [3] are considered. The DEMUX/MUX crosstalk can be reduced by placing narrow-band optical filters before multiplexing. The amount of suppression of the inter-band components will depend on the transfer function $T(\Delta \lambda)$ of the filters.

Crosstalk varies with the traffic parameters of the network; i.e., generation (g), absorption, link utilization probabilities, probability mass function of the age of the packets, architecture of the submodules and routing algorithm [4].

\section{RESULTS}

We analyzed a network with four $2.5 \mathrm{~Gb} / \mathrm{s}$ channels in the range of $1550 \mathrm{~nm}$ to $1556 \mathrm{~nm}$, with 2 nm channel separation. DEMUX with adjacent signal inter-band crosstalk of $-30 \mathrm{~dB}$, and a $2 \times 2$ crossbar optical switch with coupling power coefficient $\alpha$ between $-25 \mathrm{~dB}$ and $-35 \mathrm{~dB}$ are assumed. Filters have a transfer function $T(\Delta \lambda)=-17 \mathrm{~dB}$. We represent each amplifier by using the spectrally resolved numerical model of [5] with a forward pumping scheme. The absorption and gain parameters are the same as those of fiber 2a in [6] with a length of $20 \mathrm{~m}$ and a pump power of $50 \mathrm{~mW}$. A bandwidth of $125 \mathrm{GHz}$ is used to resolve the effect of ASE spectrum. The optical filter at the receiver has a $0.2 \mathrm{~nm}$ bandwidth and the electrical filter has a $2.5 \mathrm{GHz}$ bandwidth. We assumed a fiber with dispersion coefficient $D_{c}=1 \mathrm{ps} / \mathrm{km}-\mathrm{nm}$, a loss coefficient of $0.2 \mathrm{~dB} / \mathrm{km}$, an inter-node distance of $15 \mathrm{~km}$, a total node loss of $12.5 \mathrm{~dB}$, for hot-potato and $15.5 \mathrm{~dB}$ for single-buffer node architectures. The optical amplifiers are located at the output of each node.

We optimized the performance of the network based on the input power. Thus, Fig. 2 shows the maximum number of nodes $n$ traversed by a signal with a $\operatorname{BER}(\mathrm{n})<10^{-9}$ versus input power at $\mathrm{g}=\mathbf{1}$ for the channel at $1556 \mathrm{~nm}$, one with the worst gain. Results are shown for Hot-potato under SN topology with $\alpha=-35 \mathrm{~dB}$ and $-25 \mathrm{~dB}$. For low input power the predominant beat noise is signal-ASE that increases with bit rate and for high input powers the signalcrosstalk beat dominates, a noise that is bit rate independent.

Fig. 3 shows results for the conditional $\operatorname{BER}(n)$ versus number of nodes $n$ traversed by a packet when $g=0.1$ and $g=1$ for all the submodules of a network 


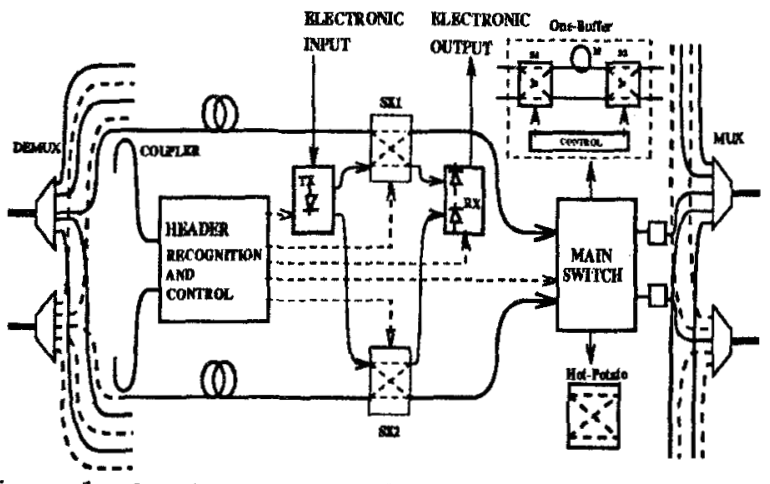

Figure 1: Node block diagram, Hot-potato switch, and Onebuffer switch, $M$ is the memory, $S 1$ and $S 2$ are exchange-bypass switches.

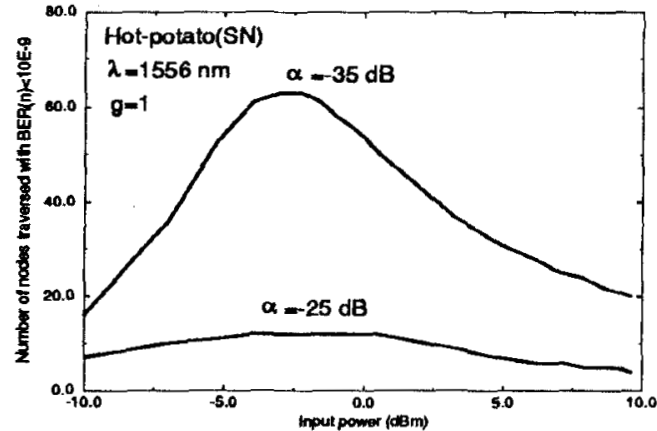

Figure 2: Maximum number of nodes traversed by a signal with a $\operatorname{BER}(n)<10^{-9}$ versus input power at $\mathrm{g}=1$.

with 64 nodes. Observe that SN and MS have a very similar BER(n) performance. The reason is that the link utilization probability and the average age of the packets of both topologies are similar [4]. Observe that the BER(n) degrades at full load $(\mathrm{g}=1)$. This is because there are more packets in the network and the crosstalk probability increases. The two main reasons why single-buffer performs worse than hot-potato are: Single-buffer has two optical switches to route the packets, therefore more intraband crosstalk; the second is that ASE noise increases because the EDFA's gain increases trying to compensate the loss introduced by the the addition of optical switches. Although using optical buffers deteriorates the conditional BER(n), a fair comparison should give curves of average BER vs throughput, as in Fig. 4. Observe that at $\mathrm{BER}=10^{-9}$ single-buffer has a higher throughput [1] than hot-potato. Also, observe that under the same throughput SN has a better BER performance than MS due to the fact that SN has less traffic congestion.

\section{CONCLUSIONS}

Crosstalk and ASE noise limit the propagation distance, traffic load and bit rate performance of the network. Results show that hot-potato deflection routing has a better BER performance than single-buffer

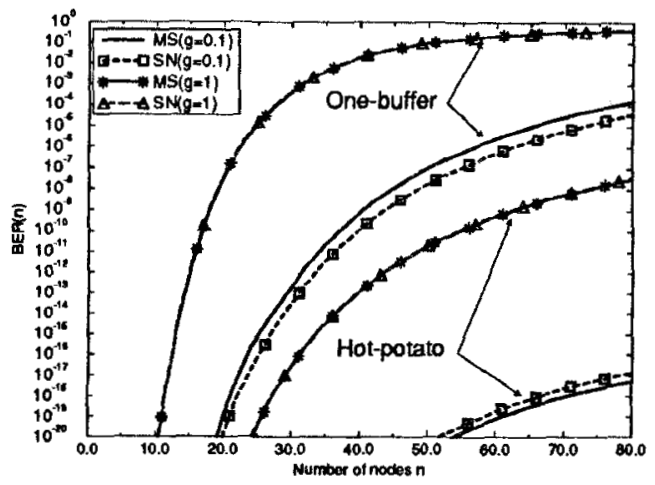

Figure 3: $B E R(n)$ versus number of nodes for $g=0.1$ and $\mathrm{g}=1$. Curves are for wavelength $1556 \mathrm{~nm}$

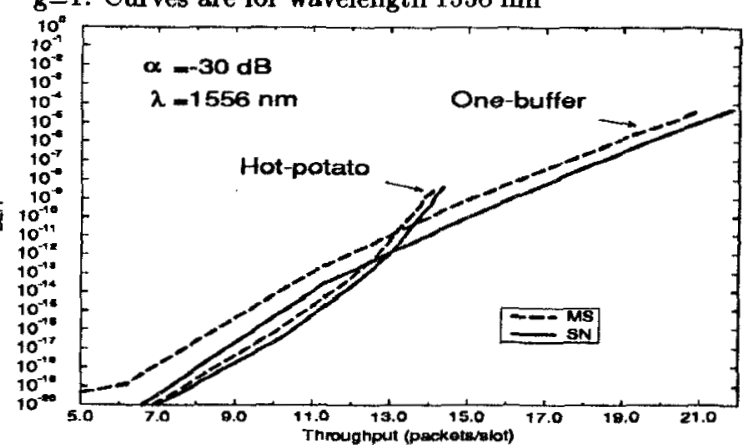

Figure 4: BER versus throughput for networks with 64 nodes for $g=1$ and $\alpha=-30 \mathrm{~dB}$.

under low throughput. However, at BER $=10^{-9}$ singlebuffer has a higher throughput than hot-potato due to the fact that single-buffer has less traffic congestion. If the bit rate increases the BER performance is worse than the shown, but if it decreases the BER improves making possible the operation of single-buffer at high throughput. The feasibility of deflection routing transparent networks will depend on the isolation characteristics of the optical switching elements. The most crucial component is the routing space switch. A high coupling power coefficient $\alpha$ limits the operation of the network, while a low $\alpha$ will make its operation possible with good performance.

\section{References}

[1] F. Forghieri, A. Bononi and P.R. Prucnal, IEEE Trans. on Commun., Vol. 43, No. 1, pp. 88-98, January 1995.

[2] A. Bononi, F. Forghieri, and P.R. Prucnal, IEEE J. Lightwave Technol, Vol. 11, No. 12, pp. 2166-2176, Dec 1993.

[3] J. Zhou, R. Cadeddu, E. Casaccia, C. Cavazzoni, and M. J. O'Mahony, JEEE J. Lightwave Technol, to appear.

[4] G. A. Castanon, O. K. Tonguz, and A. Bononi, IEE Proc.Communications, submitted for publication.

[5] C. R. Giles and E. Desuvire, J. Lightwave Technol., Vol. 9, No. 2, pp. 271-283, Feb. 1991.

[6] C. R. Giles, C. Burrus, D. DiGiovanni, N. Dutta, and G. Raybon, IEEE Photon. Technol. Lett., Vol. 3, pp. 363365,1991 . 